\title{
Exosomal microRNA-144 from bone marrow-derived mesenchymal stem cells inhibits the progression of non-small cell lung cancer by targeting CCNE1 and CCNE2
}

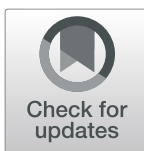

Yuan Liang ${ }^{1}$, Dalin Zhang ${ }^{2}$, Linlin Li ${ }^{1}$, Tian Xin ${ }^{1}$, Yuwei Zhao ${ }^{1}$, Rui Ma ${ }^{1 *}$ (D) and Jiang Dü ${ }^{3 *}$

\begin{abstract}
Background: Mesenchymal stem cells (MSCs) are pluripotent mesenchymal cells present in various adult tissues. MSCs secrete exosomes as regulators of the tumor niche, with involvement in tumorigenesis and metastasis. The regulatory role of microRNAs (miRs or miRNAs) in MSCs via targeting cyclin E1 (CCNE1) or cyclin E2 (CCNE2) has been extensively reported. Since exosomes are considered as protective and enriched sources of shuttle miRNAs, we hypothesized that exosomal transfer of miR-144 from bone marrow-derived MSCs (BMMSCs) would affect the development of non-small cell lung cancer (NSCLC) cells by targeting CCNE1 and CCNE2.
\end{abstract}

Methods: We first quantified the levels of miR-144, CCNE1, and CCNE2 in NSCLC tissues and cell lines and then undertook gain- and loss-of-function studies of miR-144, CCNE1, and CCNE2 to investigate their roles in the biological characteristics of NSCLC in vitro. NSCLC cells (A549) were exposed to exosomes derived from MSCs, and cell proliferation and colony formation rate were determined using in vitro assays. Finally, effects of BMMSC-derived exosomal miR-144 on tumor development were studied in vivo.

Results: In NSCLC tissues and cell lines, miR-144 was expressed poorly and CCNE1 and CCNE2 were expressed highly. Artificially elevating miR-144 inhibited cell proliferation, colony formation, and the number of S phasearrested cells in NSCLC by downregulating CCNE1 and CCNE2. Additionally, BMMSC-derived exosomal miR-144 led to restrained NSCLC cell proliferation and colony formation. These inhibitory effects of BMMSC-derived exosomes carrying miR-144 on NSCLC were confirmed by experiments in vivo.

Conclusion: Collectively, these findings revealed inhibitory effects of BMMSC-derived exosomal miR-144 on NSCLC progression, which were mediated by downregulation of CCNE1 and CCNE2.

Keywords: Non-small cell lung cancer, Bone marrow-derived mesenchymal stem cells, Exosomes, MicroRNA-144, Cyclin E1, Cyclin E2

\footnotetext{
* Correspondence: marui2222@sina.com; jdu@cmu.edu.cn

${ }^{1}$ Medical Oncology Department of Thoracic Cancer (2), Cancer Hospital of China Medical University, Liaoning Cancer Hospital \& Institute, No. 44, Xiaoheyan Road, Dadong District, Shenyang 110042, Liaoning Province, People's Republic of China

${ }^{3}$ Department of Pathology, The First Affiliated Hospital and College of Basic

Medical Science, China Medical University, No. 155, Nanjing North Street,

Heping District, Shenyang 110001, Liaoning Province, People's Republic of

China

Full list of author information is available at the end of the article
}

(c) The Author(s). 2020 Open Access This article is distributed under the terms of the Creative Commons Attribution 4.0 International License (http://creativecommons.org/licenses/by/4.0/), which permits unrestricted use, distribution, and reproduction in any medium, provided you give appropriate credit to the original author(s) and the source, provide a link to the Creative Commons license, and indicate if changes were made. The Creative Commons Public Domain Dedication waiver (http://creativecommons.org/publicdomain/zero/1.0/) applies to the data made available in this article, unless otherwise stated. 


\section{Background}

Statistical analysis from the American Cancer Society and the Cancer Statistics Center shows that each year more than 150,000 patients die from lung cancer, and 200,000 new cases are diagnosed [1]. Primary lung cancer is traditionally classified into small cell lung cancer and non-small cell lung cancer (NSCLC) [2]. Surgical resection is the main treatment method for lung cancer, but most patients are diagnosed at the advanced disease stage, when relatively ineffective drug therapy is the only feasible treatment [2]. Mesenchymal stem cells (MSCs) are non-hematopoietic progenitor cells that can be obtained from bone marrow aspirates or adipose tissue, and then expanded and genetically modified in vitro, presenting a potential therapeutic strategy for cancer patients [3]. The molecular mechanisms behind the function of MSCs in tumorigenesis and cancer development are not well-defined, but better knowledge of these mechanisms could facilitated improved prognosis and suppressed malignancy.

Cyclin E1 (CCNE1) is an important factor that can regulate the entry of proliferating cells into $\mathrm{S}$ and G1 phases, which is reported to play a role in regulating growth of lung cancer cells [4]. A previous study revealed that the cancer cell cycle progression was inhibited by ZYG-11 family member A (ZYG11A), which targets CCNE1 [5]. Meanwhile, downregulation of cyclin E2 (CCNE2) is demonstrated to notably restrain NSCLC cell function [6]. Overexpression of CCNE2 antagonizes the inhibitory effect of microRNA (miR)-30d-5p on the proliferation and motility abilities of NSCLC cells [6]. Collectively, the miRNAs are a class of small non-coding RNA molecules consisting of 20-23 nucleotides, which plays crucial roles in many biological processes by binding to certain target mRNAs, thus restraining their translation or degradation [7]. Numerous reports have indicated that miRNAs are dysregulated in a variety of tumors and might therefore be used as plasma diagnostic markers for cancers [8]. It was previously reported that inhibition of miR-144 may enhance the metastasis of NSCLC [9].

Exosomes are small lipid bilayer-enclosed particles with a diameter of $30-140 \mathrm{~nm}$, which originate in the endosomes [10] and are released from various cell types under specific physiological or pathological conditions. Exosomes can transport multiple functional molecules, including mRNAs and miRNAs [11]. They have been identified as mediators of interactions among cancer cells and can function as long range signal effectors potentially coordinating tumor formation, progression, metastasis, drug resistance, tube formation, and tumor immunity [12]. We now test the hypothesis that BMMSC-derived exosomes carrying miR-144 can affect NSCLC growth and progression by targeting CCNE1 and CCNE2.

\section{Methods \\ Ethics statement}

This experiment was approved by the Ethics Committee of Cancer Hospital of China Medical University. All participants enrolled in the study signed informed written consent documents, and the experiment procedures were in line with the Declaration of Helsinki. All animal experiments were in strict accordance with the recommendations in the Guide for the Care and Use of Laboratory Animals of the National Institutes of Health.

\section{Microarray-based gene expression profiling}

Gene expression profiling (GSE74706, GSE33532) and miRNA profiling (GSE102286) associated to NSCLC were retrieved from the Gene Expression Omnibus database. We used the limma package of $\mathrm{R}$ language to screen the differentially expressed genes (DEGs) and miRNAs and the DAVID package for functional enrichment analysis of DEGs. The ggplot2 package in $\mathrm{R}$ language was utilized for correlational analysis of gene expression correlation and for visualization. The potential regulatory relationship between DEGs and miRNAs was predicted using the microRNA.org, miRDB, TargetScan, mirDIP, and DIANA databases. We calculated Venn diagrams to identify the overlap between different genes and miRNA data in the database.

\section{Patient enrollment}

Sixty-eight patients (46 males and 22 females, $57.28 \pm$ 4.58 years old) diagnosed with NSCLC at the Cancer Hospital of China Medical University were enrolled in this study. None of the patients had received local or systemic chemoradiotherapy before their surgery. Resected tumor tissues and adjacent normal tissues (5 $\mathrm{cm}$ away from tumor tissues) were collected from each patient and immediately stored in liquid nitrogen. Bone marrow specimens were also collected from three inpatients ( 2 males and 1 female) with osteonecrosis of the femoral head, aged from 26 to 52 years, without loss of height of the femoral head or diseases such as trauma, cardiovascular disease, or tumor invasion according to magnetic resonance imaging examinations.

\section{Immunohistochemistry (IHC)}

The paraffin-embedded tissues were sliced, dewaxed, dehydrated with gradient ethanol, and immersed in $3 \% \mathrm{H}_{2} \mathrm{O}_{2}$. Next, the sections were subjected to 
antigen retrieval and then blocked with normal goat serum (C-0005, Shanghai Haoran Bio Technologies Co., Ltd., Shanghai, China) for $20 \mathrm{~min}$. The sections were subsequently incubated with primary antibody mouse anti-CCNE1 (1:100, ab238081), CCNE2 (1:50, ab32103), and KI-67 (1:150, ab156956) at $4{ }^{\circ} \mathrm{C}$ overnight. The sections were further incubated with secondary antibody goat anti-mouse immunoglobulin G (IgG) (1:1000, ab6785, Abcam Inc., Cambridge, MA, UK) at $37^{\circ} \mathrm{C}$ for $20 \mathrm{~min}$, followed by incubation with peroxidase horseradish-labeled streptavidin ovalbumin solution at $37^{\circ} \mathrm{C}$ for $20 \mathrm{~min}$. After being developed with diaminobenzidine, the sections were counterstained by hematoxylin (PT001, Shanghai Bogoo Biotechnology., Co., Ltd., Shanghai, China) for $1 \mathrm{~min}$, blued with ammonia water, and then dehydrated with gradient ethanol, cleared by xylene, sealed by neutral balsam, and observed under a microscope.

\section{Cell treatment}

NSCLC cell lines A549, NCI-H1975, NCI-H1299, and SPC-A1 and normal human bronchial epithelial (HBE) cell were purchased from Bena Culture Collection (Suzhou, China). The cells were cultured in Dulbecco's modified Eagle's medium (DMEM; 12800017, Gibco, Carlsbad, CA, USA) containing 10\% fetal bovine serum (FBS, 26140079, Gibco, Carlsbad, CA, USA) at $37^{\circ} \mathrm{C}$ with $5 \% \mathrm{CO}_{2}$ and saturated humidity. The cells $\left(1 \times 10^{5}\right.$ cells $/ \mathrm{mL}$ ) were seeded into 6-well plates and cultured for $24 \mathrm{~h}$. Cells were then transfected with plasmids of miR144 mimic, miR-144 inhibitor, short hairpin (sh)CCNE1, sh-CCNE2, or their relative negative control (NC) individually or together, using Lipofectamine 2000 reagent (Invitrogen Inc., Carlsbad, CA, USA) according to the manufacturer's instructions. The plasmids of shNC, sh-CCNE1, and sh-CCNE2 were purchased from Guangzhou RiboBio Co., Ltd. (Guangzhou, Guangdong China), and plasmids of mimic-NC, miR-144 mimic, inhibitor-NC, and miR-144 inhibitor were from Shanghai GenePharma Co., Ltd. (Shanghai, China). BMMSCs and NSCLC cells were transfected by Lipofectamine 2000. Then, a 250- $\mu \mathrm{L}$ volume of Opti-minimum essential medium (MEM) (Gibco, Grand Island, NY, USA) was used to dilute $4 \mu \mathrm{g}$ target plasmids and $10 \mu \mathrm{L}$ Lipofectamine 2000 respectively. After mixing by gentle shaking, the samples were allowed to stand for $5 \mathrm{~min}$ at room temperature. Next, the two liquids were mixed for $20 \mathrm{~min}$ of reaction and then added to the cell culture wells. The culture plate was shaken, and then placed in a $5 \% \mathrm{CO}_{2}$ incubator at $37^{\circ} \mathrm{C}$ for further culture. After $6 \mathrm{~h}$, the culture medium was changed, and cells were collected after $36-48 \mathrm{~h}$ of transfection for subsequent experiments.
Table 1 Primer sequences for RT-qPCR

\begin{tabular}{ll}
\hline Gene & Primer sequence (5'-3') \\
\hline miR-144 & F: 5'-TCCGATCATGTAGTAGATATTGACAT-3' \\
& R: 5'-GTGCAGGGTCCGAGGT-3' \\
CCNE1 & F: 5'-GTGGCTCCGACCTTTCAGTC-3' \\
& R: 5'-CACAGTCTTGTCAATCTTGGCA-3' \\
CCNE2 & F: 5'-GCATTATGACACCACCGAAGA-3' \\
& R: 5'-TAGGGCAATCAATCAATCACAGC-3' \\
U6 & F: 5'-TCTTGGAATTCAAGGTCGGGCAGGAAGAGGGCCTA-3' \\
& R: 5'-CGCGGATCCTAGTATATGTGCTGCCGAAGC-3' \\
GAPDH & F: 5'-GAAGGTGAAGGTCGGAGT-3' \\
& R: 5'-GAAGATGGTGATGGGATTC-3'
\end{tabular}

$\mathrm{RT}$-qPCR, reverse transcription quantitative polymerase chain reaction; $F$ forward, $R$ reverse, miR-144 microRNA-144, CCNE1 cyclin E1, CCNE2 cyclin E2, GAPDH glyceraldehyde-3-phosphate dehydrogenase

\section{Reverse transcription quantitative polymerase chain reaction ( $\mathrm{RT}$-qPCR)}

The total RNA was extracted using a Trizol Kit (15596026, Invitrogen, Carlsbad, CA, USA) and reversely transcribed into complementary DNA by PrimeScript RT reagent Kit (RR047A, Takara Bio Inc., Otsu, Japan). RT-qPCR was then performed with Fast SYBR Green PCR kit (Applied Biosystems, Carlsbad, CA, USA) on an ABI 7500 instrument (Applied Biosystems, Carlsbad, CA, USA). The relative expression of genes was calculated by means of relative quantification (2 $2^{-\triangle \mathrm{Ct}}$ method) with glyceraldehyde-3phosphate dehydrogenase (GAPDH) and U6 as internal controls. The primer sequences are shown in Table 1.

\section{Western blot analysis}

The total protein content was isolated with an enhanced radio immunoprecipitation assay lysis buffer (Wuhan Boster Biological Technology Co., Ltd., Wuhan, China). The proteins were separated by sodium dodecyl sulfatepolyacrylamide gel electrophoresis and then transferred to a polyvinylidene fluoride membrane. After being blocked in sealing solution, the membrane was incubated with the primary antibodies rabbit anti-human CCNE1 (1:2000, ab33911), CCNE2 (1:500, ab32103), KI67 (1: 000, ab92742), proliferating cell nuclear antigen (PCNA) (1:1000, ab925522), or GAPDH (1:5000, ab181602, all from Abcam Inc., Cambridge, MA, USA), which served as a $\mathrm{NC}$, at $4{ }^{\circ} \mathrm{C}$ overnight. The next day, the membrane was incubated with secondary goat antirabbit IgG (1:10000, ab205718, Abcam Inc., Cambridge, MA, USA) at $37^{\circ} \mathrm{C}$ for $1 \mathrm{~h}$. The samples were developed using ECL reaction solution, photographed using SmartView Pro 2000 (UVCI-2100, Major Science, Saratoga, 
CA, USA), followed by gray scale analysis of the protein band pattern using the Quantity One software.

\section{Dual luciferase reporter assay}

The 3' untranslated regions (UTRs) of CCNE1 and CCNE2, which contain potential miR-144 binding sites, were constructed into the PGLO vector (PGLOCCNE1 wild type (WT) and PGLO-CCNE2 WT). The mutant (MUT) forms, in which the potential miR-144 binding sites were mutated for loss of function, were also constructed (PGLO-CCNE1 MUT and PGLOCCNE2 MUT). Report plasmids were co-transfected with miR-144 mimic, or miR-NC into HEK293T cells. After $24 \mathrm{~h}$ of transfection, the cells were lysed and centrifuged, and the supernatant was collected. The luciferase activity was detected using Dual-Luciferase ${ }^{\circ}$ Reporter Assay System (E1910, Promega Corp., Madison, WI, USA) according to the manufacturer's instructions.

\section{Isolation and identification of BMMSCs}

BMMSCs were isolated from the three bone marrow donations as previously reported [13] and cultured in DMEM-F12 (Hyclone, South Logan, UT, USA) containing 10\% FBS (10099141, Gibco, Carlsbad, CA, USA) and $0.2 \%$ penicillin and streptomycin (Hyclone, South Logan, UT, USA). Then, the cells were passaged every 3 days, and BMMSCs of the third to seventh passages were used for further experiments. The BMMSCs were cultured in BMMSCs' osteogenic, adipogenic, and cartilagedifferentiated OriCell ${ }^{\mathrm{Th}}$ medium (Cyagen Biosciences Inc., Guangzhou, China). Finally, the BMMSCs were stained with alizarin red and oil red $\mathrm{O}$.

BMMSCs at the third passage were incubated with mouse monoclonal antibodies against CD105 (ab11414, 1:100), CD73 (ab81720, 1:50), CD90 (ab23894, 1:100), CD45 (ab8216, 1:50), CD34 (ab8536, 1:50), CD14 (ab182032, 1:200), CD19 (ab31947, 1:50), HLA-DR (ab20181, 1:50), and goat anti-mouse IgG isotope antibody (1:1000, BD Biosciences Pharmingen, San Jose, CA, USA) conjugated with fluorescein isothiocyanate (FITC). The above antibodies were supplied by Abcam Inc. (Cambridge, MA, UK). The samples were analyzed with the FACSVerse instrument (BD Biosciences Pharmingen, San Jose, CA, USA) with FlowJo software (Tree Star Inc., Ashland, OR, USA).

\section{Isolation and identification of BMMSC-derived exosomes}

The BMMSCs at the logarithmic growth phase were collected, and their secreted exosomes were isolated from the supernatant by gradient centrifugation. The protein concentration of exosomes was determined by the bicinchoninic acid (BCA) assay. Expression of specific surface biomarkers of exosomes (CD63, CD81,
TSG101, and calnexin) was detected immunohistochemically. Zetasizer Nano ZS (Malvern Panalytical Ltd., Malvern, UK) was used to determine the particle size of exosomes. The exosome suspension solution was fixed with $2 \%$ paraformaldehyde, $2.5 \%$ glutaraldehyde, and $1 \%$ osmic acid for $1.5 \mathrm{~h}$. The fixed exosomes were dehydrated with gradient ethanol, immersed in epoxy resin overnight, and polymerized at 35,45 , and then $60^{\circ} \mathrm{C}$ for $24 \mathrm{~h}$. Lastly, the embedded exosomes were cut into ultrathin slices, stained with lead and uranium salts, and observed under a transmission electron microscope.

\section{Labeling and tracking of BMMSC-derived exosomes}

According to the manufacturer's protocol, BMMSCderived exosomes were labeled using CM-Dil (Beyotime Biotechnology Inc., Haimen, China), and then cultured at $37^{\circ} \mathrm{C}$ for $30 \mathrm{~min}$ in the dark. To remove unbound dye, the exosomes were rinsed with phosphate-buffered saline (PBS) and centrifuged at $100,000 \times g$ at $4{ }^{\circ} \mathrm{C}$ for 70 $\mathrm{min}$ and then at $800 \times \mathrm{g}$ for $5 \mathrm{~min}$ at room temperature. At last, the exosomes were mixed together and incubated at $37^{\circ} \mathrm{C}$ for $24 \mathrm{~h}$. The uptake of dye by exosomes was then observed under a fluorescence microscopy (Leica, Weltzlar, Germany), with image analysis using Leica Application Suite Advanced Fluorescence software.

\section{Co-culture of BMMSC-derived exosomes and NSCLC cells}

Exosomes were extracted from transfected BMMSCs, and then co-cultured with NSCLC cells transfected with NC mimic, miR-144 mimic, NC inhibitor, or miR-144 inhibitor for $48 \mathrm{~h}$.

\section{5-Ethynyl-2'-deoxyuridine (EdU) assay}

Cells were seeded into 24-well plates, with triplicate wells for each group. Subsequently, the cultured cells were added with EdU (C10341-1, Guangzhou RiboBio Co., LTD., Guangzhou, China) to a final concentration of $10 \mu \mathrm{M}$, with culture continuing for another $2 \mathrm{~h}$. Next, the cells were fixed, rinsed, and then incubated with PBS containing $0.5 \%$ Triton-100. Suspended cells were then stained with Apollo 567 (Guangzhou RiboBio Co., LTD, Guangzhou, China) for $30 \mathrm{~min}$ and with $1 \times$ Hoechst 33342 for $30 \mathrm{~min}$. Finally, the number of positive cells was recorded under a fluorescence microscope (model: FM-600, Shanghai Pudan Optical Instrument Co., Ltd., Shanghai, China).

\section{Colony formation assay}

Cells were seeded in $10 \mathrm{~mL}$ culture medium (200 cells/culture dish) and cultured for 3 weeks. When visible clones were observed, cells were fixed and stained with $0.1 \%$ crystal violet for $10 \mathrm{~min}$. Finally, the 
number of clones was counted, while the colony formation rate was calculated as follows: colony formation rate $=$ the number of clones/the number of plated cells $\times 100 \%$.

\section{Flow cytometry}

Cells $\left(1 \times 10^{6}\right.$ cells $\left./ \mathrm{mL}\right)$ were fixed with $70 \%$ precooled ethanol at $4{ }^{\circ} \mathrm{C}$ overnight. Next, $100 \mu \mathrm{L}$ portions of cell suspension was stained with $50 \mu \mathrm{g}$ propidium iodide (PI) dye liquor (40710ES03, Shanghai Qcbio Science \& Technologies CO., Ltd., Shanghai, China) containing ribonuclease at $4{ }^{\circ} \mathrm{C}$ for $30 \mathrm{~min}$ in the dark. Finally, cell cycle was recorded by detecting (red) fluorescence at $488 \mathrm{~nm}$ in conjunction with flow cytometry (BD, FL, NJ, USA) after filtering of the cell suspension through 100 mesh nylon.

\section{Xenograft tumor model}

Fifteen BALB/c-nu female nude mice (5-6 weeks, 18 $22 \mathrm{~g}$ ) were randomly allocated to three groups of five animals each. NSCLC cell suspension $\left(1 \times 10^{7}\right.$ cells/ mouse) was subcutaneously injected into each mouse to establish the subcutaneous xenograft tumor model. When the mean tumor volume reached $100 \mathrm{~mm}^{3}$, the BMMSC-secreted exosomes $(100 \mu \mathrm{L}$, concentration of $1 \mu \mathrm{g} / \mu \mathrm{L})$ were injected into the tail vein of each mouse on the 5th, 10th, 15th, and 20th days. The injections consisted of (1) normal saline (control group), (2) exosomes carrying negative control of miR-144 agomir (Exo-NC-agomir group), and (3) exosomes carrying miR-144 agomir (Exo-miR-144 agomir group). Tumor growth (volume and weight) was observed, and tumor volume $\left(\mathrm{mm}^{3}\right)$ was calculated as $0.5 \times$ the long axis $(\mathrm{mm}) \times$ the short axis ${ }^{2}\left(\mathrm{~mm}^{2}\right)$. Twenty-five days later, all nude mice were euthanized and their tumor tissues were isolated. Histopathological analysis of the tumor tissue was performed after paraffin embedding and slicing.

\section{Statistical analysis}

Statistical analyses were performed using SPSS 21.0 statistical software (IBM Corp., Armonk, NY, USA). Measurement data were presented as mean \pm standard deviation. The paired $t$ test was applied for comparisons between paired data conforming to the homogeneity of variance and normal distribution, while the unpaired $t$ test was used for comparisons between unpaired data conforming to the homogeneity of variance and normal distribution. Data among multiple groups were analyzed by one-way analysis of variance (ANOVA), followed by a Tukey multiple comparisons post-test. Data among multiple groups at different time points were measured by repeated measures ANOVA, followed by Bonferroni post hoc test. A value of $p<0.05$ was statistically significant.

\section{Results \\ CCNE1 and CCNE2 were highly expressed in NSCLC tissues and cell lines}

The difference analysis was performed on expression profiles of NSCLC (GSE74706 and GSE33532), and 717 common DEGs were obtained based on the Venn diagram (Fig. 1a). The DEGs were enriched in signaling pathways involved in cell cycle, the p53 signaling pathway, and ECM-receptor interaction (Fig. 1b). CCNE1 and CCNE2, which belong to the cyclin family, participate in regulating cell cycle, the p53 signaling pathway, and oocyte meiosis [14]. Therefore, we investigated whether CCNE1 and CCNE2 were involved in NSCLC.

Both CCNE1 and CCNE2 proved to be highly expressed in NSCLC GSE74706 (Fig. 1c) and GSE33532 (Fig. 1d). The expressions of CCNE1 and CCNE2 were positively correlated in the expression profile of GSE74706 and GSE33532 (Fig. 1e). Levels of CCNE1 and CCNE2 in NSCLC tissues were higher than those in adjacent normal tissues $(p<0.05)$ (Fig. 1f, g). Meanwhile, IHC demonstrated that, in comparison with adjacent normal tissues, CCNE1 and CCNE2 expressions were elevated and that CCNE1 and CCNE2 were located in the nucleus $(p<0.05)$ (Fig. 1h). Furthermore, expressions of CCNE1 and CCNE2 in NSCLC cell lines (A549, NCI-H1975, NCIH1299, SPC-A1) were much higher than in normal HBE cells $(p<0.05)$. Among the four NSCLC cell lines examined, A549 cells were found to have the highest expression of CCNE1 and CCNE2 $(p<0.05)$ (Fig. 1i, j).

Silencing of CCNE1 and CCNE2 inhibited cell proliferation, colony formation, and the number of $\mathrm{S}$ phase-arrested cells in NSCLC cells

EdU assay and colony formation assay showed that A549 cells transfected with sh-CCNE1, sh-CCNE2, or co-transfected with sh-CCNE1 and sh-CCNE2 exhibited restrained abilities of proliferation and colony formation, with co-silencing CCNE1 and CCNE2 showing the strongest effect (Fig. 2a, b). The flow cytometry assay indicated that inhibiting CCNE1 or CCNE2 arrested cells at the G0/G1 phase, while notably decreasing the number of cells arrested at $\mathrm{S}$ phase $(p<0.05)$ (Fig. 2c). Expression of proliferation-related factors (KI67, PCNA) in A549 cells was reduced by sh-CCNE1 and sh-CCNE2, with the lowest expression after co-transfection with both (Fig. 2d). 

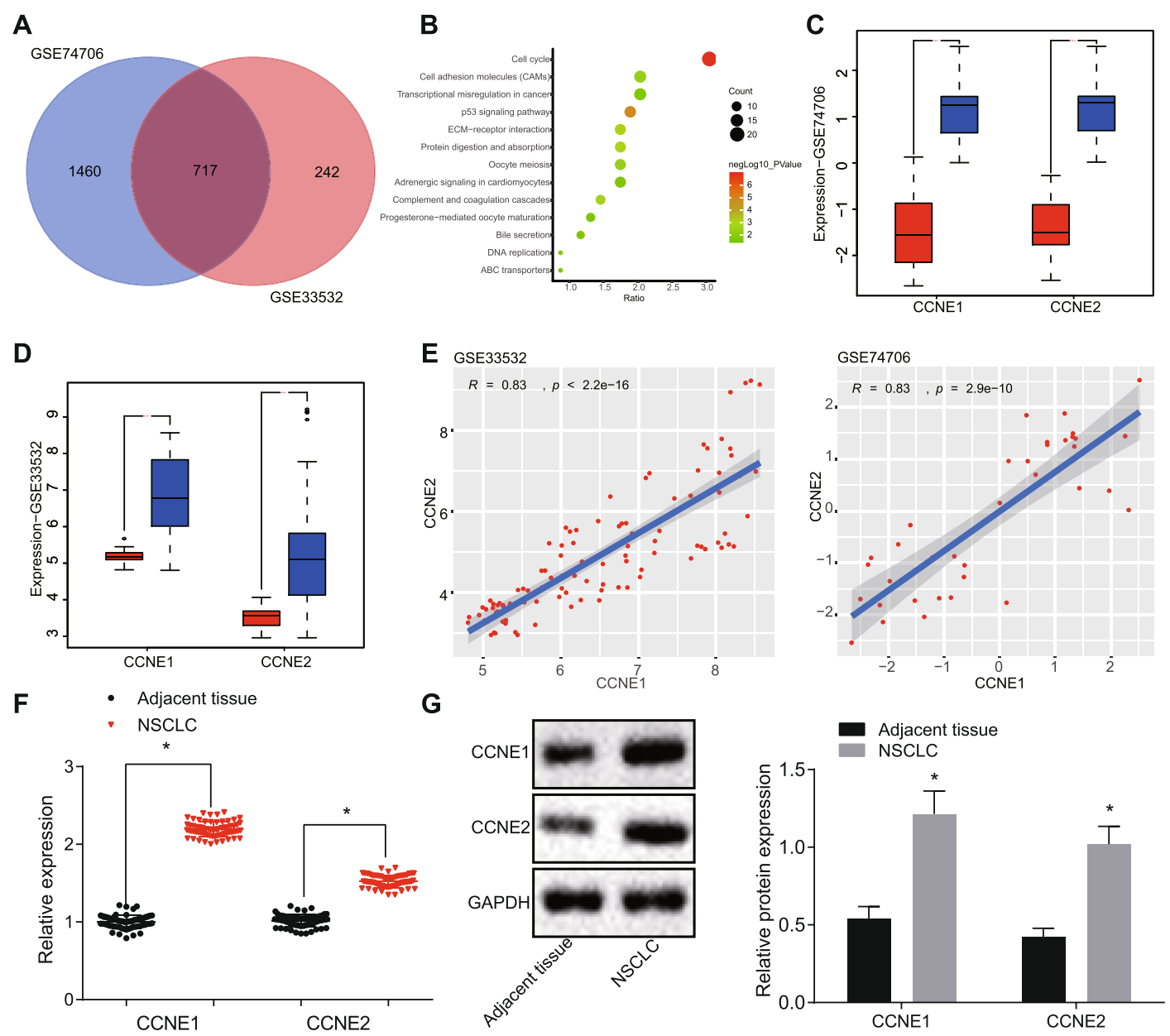

H
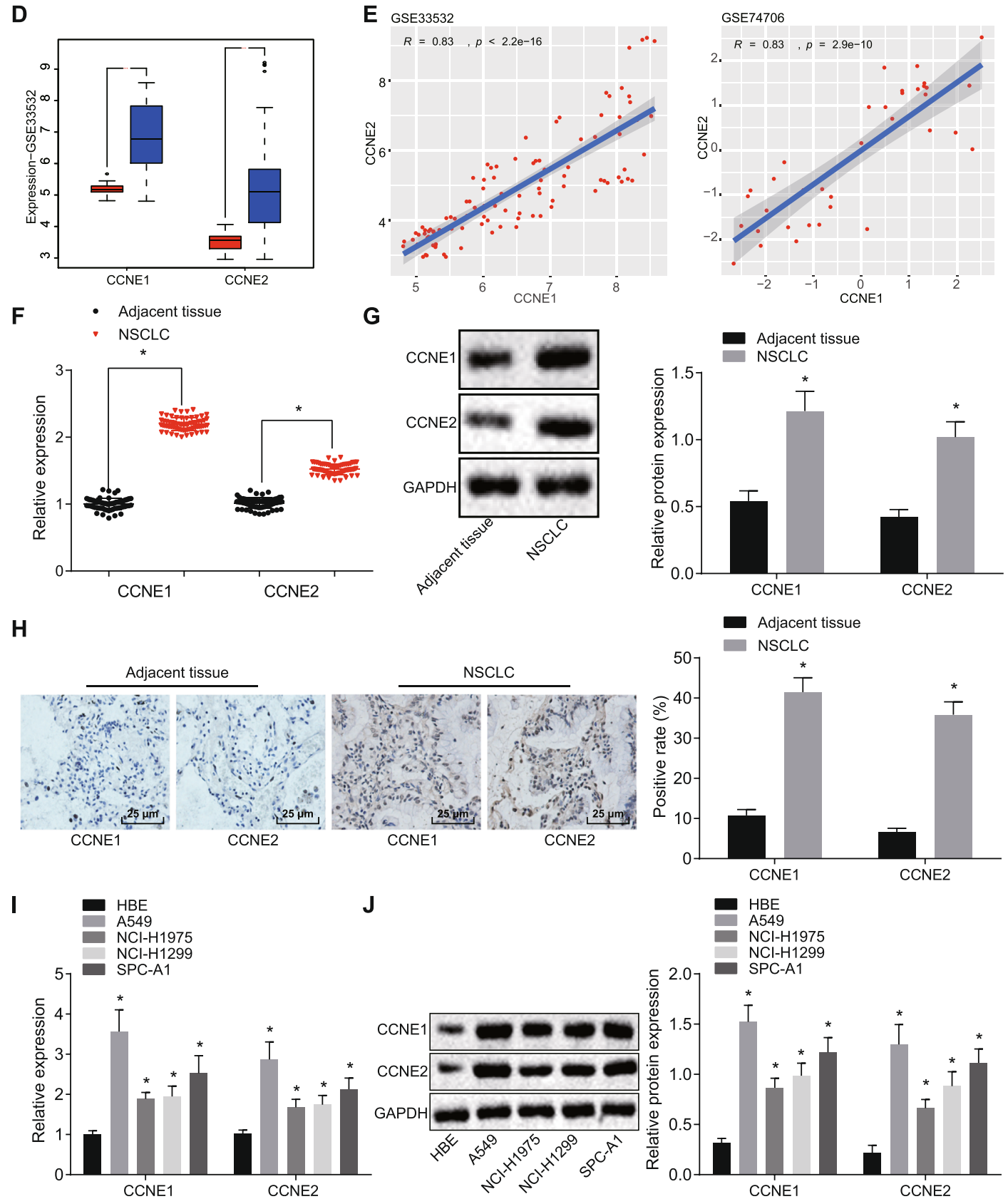

J

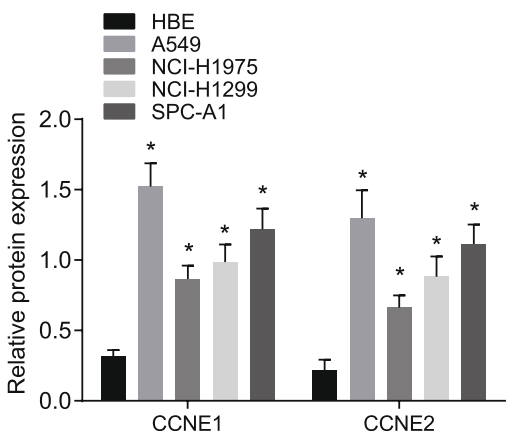

Fig. 1 (See legend on next page.) 
(See figure on previous page.)

Fig. 1 High expression of CCNE1 and CCNE2 in NSCLC tissues and cells. a Comparisons of differently expressed genes in the expression profile of NSCLCS GSE74706 and GSE33532. b KEGG pathway enrichment of the DEGs in NSCLC analyzed by DAVID software. $\mathbf{c}$, $\mathbf{d}$ Expression of CCNE1 and CCNE2 in expression profile of NSCLC GSE74706 and GSE33532. e Correlation analysis of expression of CCNE1 and CCNE2. f The expression of CCNE1 and CCNE2 in NSCLC tissues and adjacent normal tissues determined by RT-qPCR. $\mathbf{g}$ The expression of CCNE1 and CCNE2 protein in NSCLC tissues and adjacent normal tissues tested by western blot analysis. $\mathbf{h}$ Expression of CCNE1 and CCNE2 in NSCLC tissues detected by IHC $(\times 400) .{ }^{*} p<0.05$ vs. the adjacent normal tissues. $\mathbf{i}$ The expression of CCNE1 and CCNE2 in different NSCLC cell lines measured by RT-qPCR. $\mathbf{j}$ The expression of CCNE1 and CCNE2 in different NSCLC cell lines assessed by western blot analysis. ${ }^{*} p<0.05$ vs. the HBE cell line. The above measurement data were expressed as mean \pm standard deviation. Comparisons between tumor tissues and adjacent normal tissues were analyzed by paired $t$ test. Comparisons between two groups were analyzed by unpaired $t$ test. The experiment was repeated in triplicate

\section{MiR-144 is poorly expressed in NSCLC tissues and cell lines and could target CCNE1 and CCNE2}

Furthermore, upstream regulated miRNAs of CCNE1 and CCNE2 were predicted based on the databases microRNA.org, miRDB, TargetScan, mirDIP, and DIANA. The comparison of prediction results in the Venn diagram indicated nine intersecting miRNAs for CCNE1 (Fig. 3a) and 31 for CCNE2 (Fig. 3b). There was only one intersecting miRNA, hsa-miR-144-3p (Fig. 3c), suggesting that miR-144 may play a role in NSCLC by targeting CCNE1 and CCNE2. The low expression of miR-144 in GSE102286 showed that miR-144 was poorly expressed in NSCLC tissues (Fig. 3d).

In addition, RT-qPCR revealed that, compared with the adjacent normal tissues, miR-144 expression in resected NSCLC tissues was downregulated $(p<0.05)$ (Fig. 3e). RT-qPCR also revealed that, compared with normal HBE cells, the expression of miR-144 was reduced in NSCLC cell lines A549, NCI-H1975, NCIH1299, and SPC-A1, while being most significantly decreased in A549 cells $(p<0.05)$ (Fig. 3f).

The presence of specific binding sites of miR-144 on CCNE1 and CCNE2 was predicted by the bioinformatics database (http://www.microrna.org/microrna/home.do) (Fig. 3g). Then, a dual luciferase reporter assay suggested that, in contrast to transfection of $\mathrm{NC}$, the luciferase activities of WT-miR-144/CCNE1 and WT-miR-144/ CCNE2 were lowered $(p<0.05)$ (Fig. 3h). Moreover, compared with the transfection of NC mimic, expressions of CCNE1 and CCNE2 were reduced by miR-144 mimic, but upregulated by miR-144 inhibitor, compared with the transfection of NC inhibitor $(p<0.05)$ (Fig. 3i, j). Thus, miR-144 was poorly expressed in NSCLC, and miR-144 could negatively regulate expressions of CCNE1 and CCNE2.

Elevated miR-144 levels impeded cell proliferation, colony formation, and S phase-arrested cells in NSCLC cells

In contrast to findings with $\mathrm{NC}$ mimic, the proliferation and colony formation abilities were lowered by miR-144 mimic $(p<0.05)$ (Fig. $4 \mathrm{a}, \mathrm{b})$. The number of G0/G1 phase-arrested cells was increased, and $S$ phase-arrested cells were reduced in response to $\mathrm{miR}-144$ mimic treatment (Fig. 4c). Meanwhile, expression of KI67 and PCNA was notably downregulated in response to miR144 mimic $(p<0.05)$ (Fig. 4d). In conclusion, overexpression of miR-144 could lead to suppressed cell proliferation and colony formation as well as elevated G0/G1 phase-arrested cells and lowered S phasearrested cells in NSCLC cells.

\section{miR-144 suppressed NSCLC cell proliferation, colony formation, and S phase-arrested cells by targeting CCNE1 and CCNE2}

In order to test whether miR-144 regulated NSCLC progression by targeting CCNE1 and CCNE2, miR-144 was overexpressed in A549 cells with or without coexpressing CCNE1 and CCNE2. Western blot analysis indicated that the expressions of KI67 and PCNA were decreased after overexpressing miR-144 but that this effect was blocked by co-expressing of CCNE1 and CCNE2 $(p<0.05)$ (Fig. 5a).

The EdU and colony formation assays showed that miR-144 mimic could reduce cell proliferation and colony formation ability, an effect that was rescued by co-transfection of CCNE1 and CCNE2 $(p<0.05)$ (Fig. 5b, c). Cell cycle progression detected by flow cytometry revealed that the number of G0/G1 phasearrested cells was enhanced while the number of $S$ phase-arrested cells was reduced by overexpression of miR-144, an effect which could be reversed by overexpression of CCNE1 and CCNE2 at the same time $(p<0.05)$ (Fig. $5 \mathrm{~d})$. Thus, miR-144 could decrease cell proliferation, colony formation, and the number $S$ phase-arrested cells and elevate G0/G1 phase-arrested NSCLC cells by inhibiting CCNE1 and CCNE2.

\section{Characterization of BMMSC-derived exosomes}

BMMSCs were isolated from the bone marrow and subjected to flow cytometry for detection of BMMSC surface markers. As shown in Fig. 6a, the putative BMMSCs isolated from the patient's bone marrow were positive for CD105, CD73, and CD90 and 


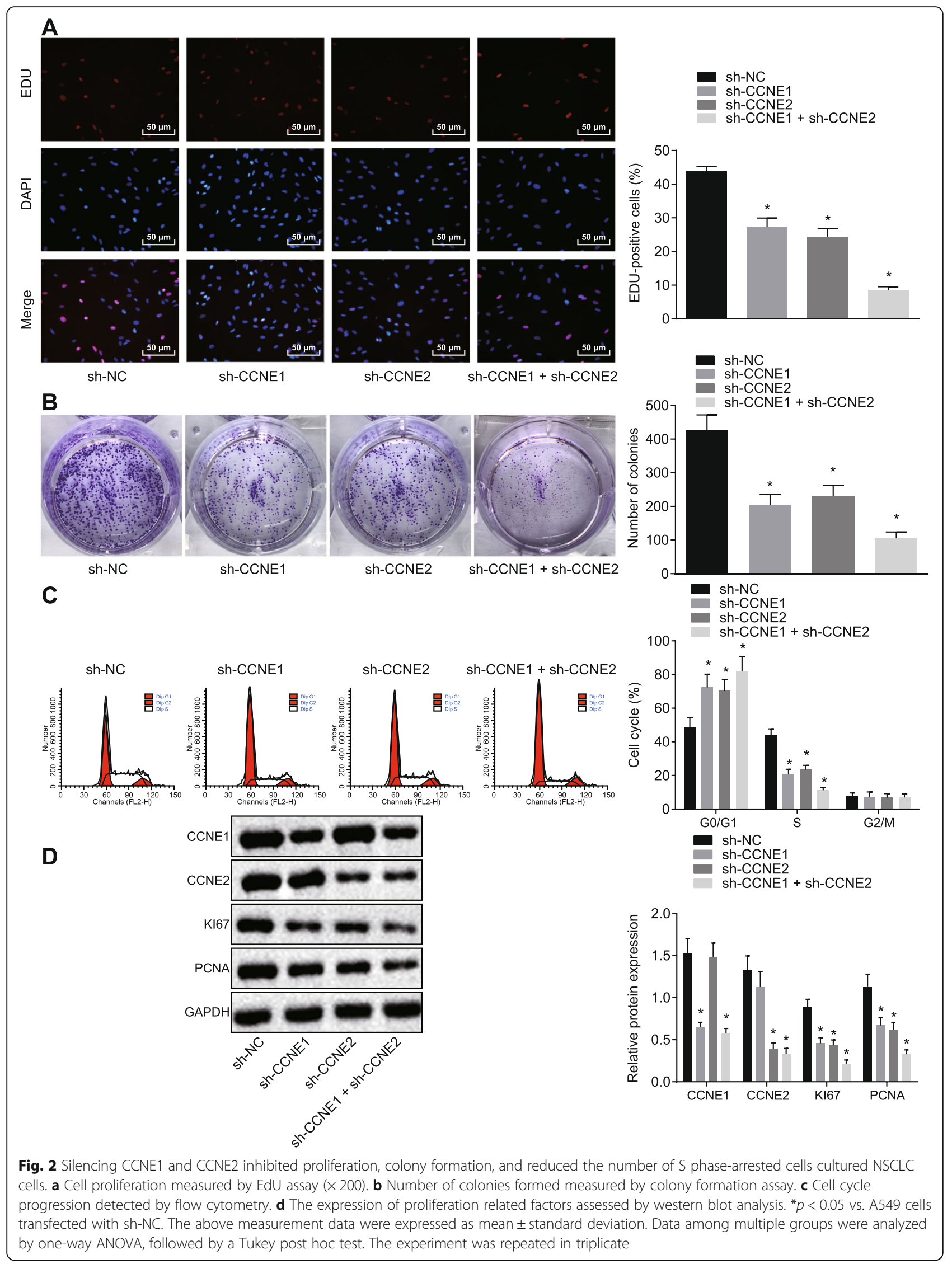




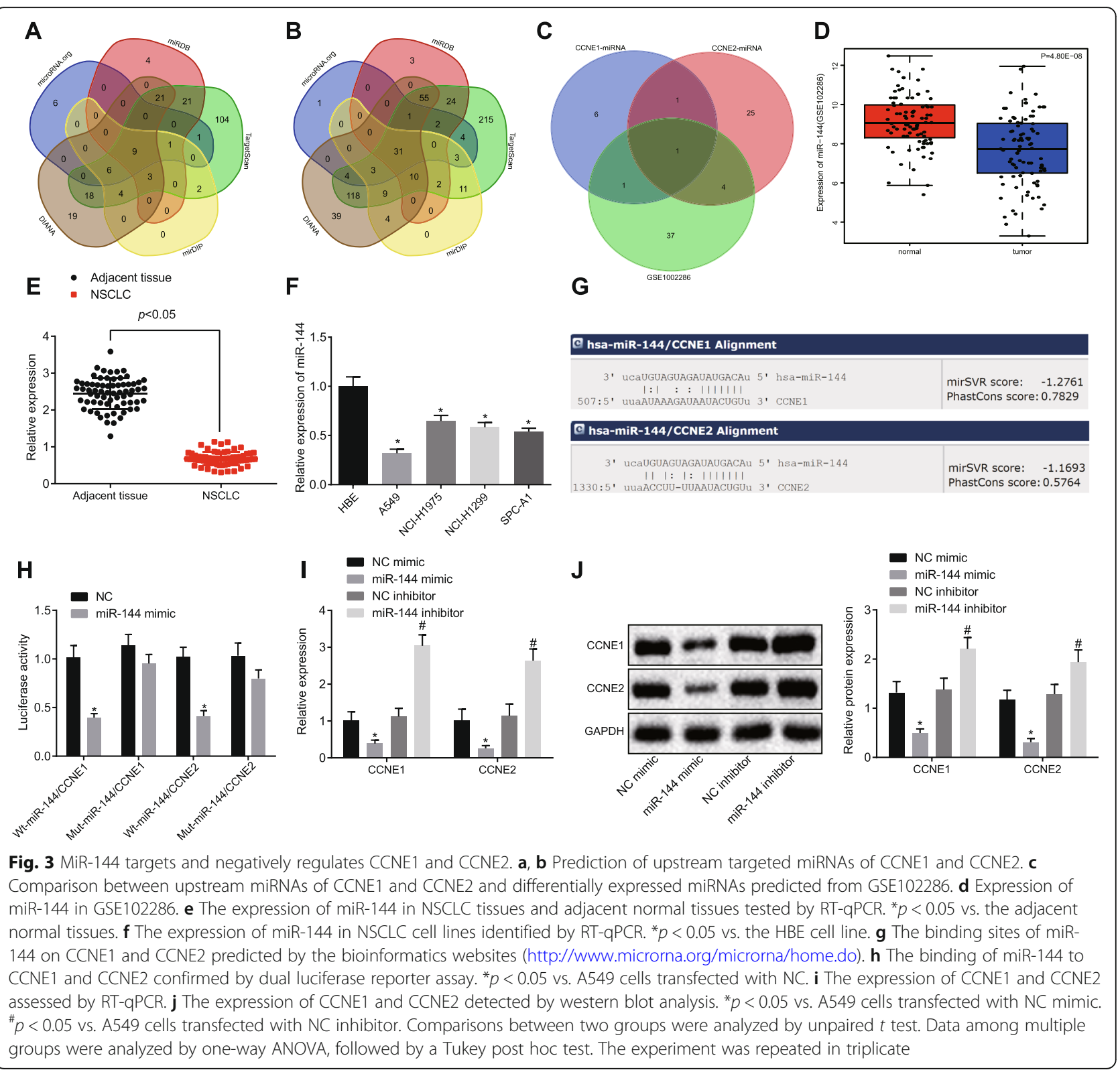

negative for CD45, CD34, CD14, CD19, and HLA-DR, thus affirming their identity as BMMSCs. The isolated BMMSCs were cultured in specific culture medium for 2 weeks to induce adipogenic differentiation, which was confirmed by the presence in the cells of red liquid droplets to oil red $\mathrm{O}$ staining (Fig. 6b). Moreover, osteogenic differentiation of BMMSCs was confirmed by presence of numerous red nodules with unclear cell structure and abundant red calcium deposition after alizarin red staining at 2 weeks after differentiation (Fig. 6c). Thus, the isolated cells were MSCs.
The exosomes isolated from BMMSCs were round and oval membranous vesicular disc structures, with intact capsule and similar morphology (Fig. 6d). Particle size of BMMSC-derived exosomes as analyzed by Zetasizer Nano ZS showed a mean diameter of approximately $62 \mathrm{~nm}$ (Fig. 6e). Expression of CD81, CD63, and TSG101 protein in BMMSC-derived exosomes was much higher than that in BMMSCs (Fig. 6f). In order to further explore the effect of BMMSC-derived exosomal miR-144 on NSCLC, we first transfected miR-144 mimic or inhibitor into BMMSCs. miR-144 expression in BMMSCs was determined by RT-qPCR (Fig. 6g), which showed that the miR- 

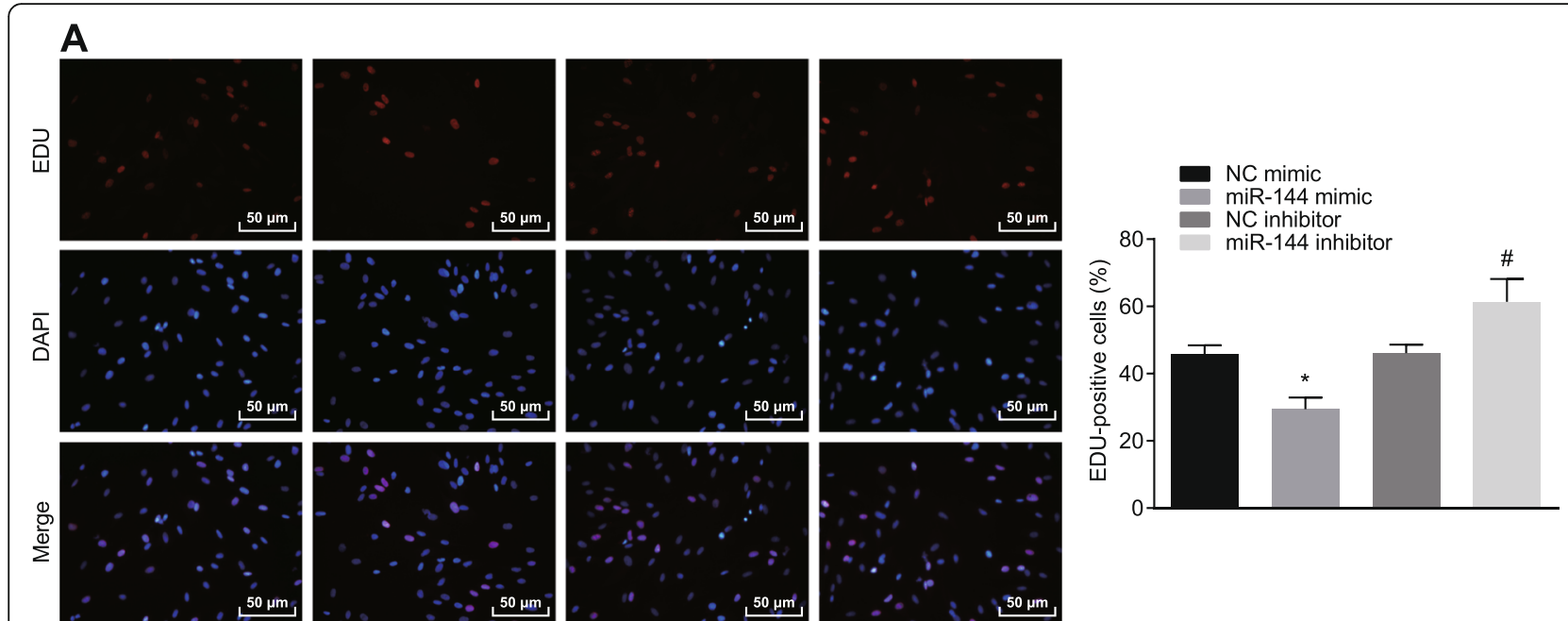

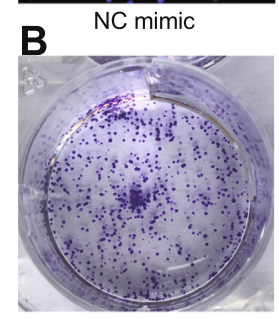

NC mimic

c
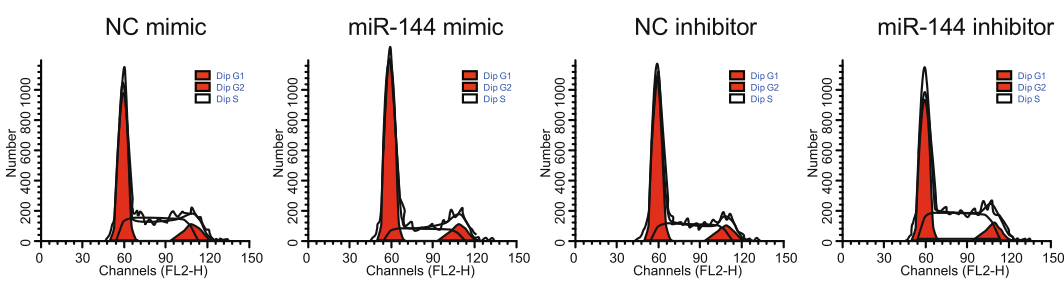

D
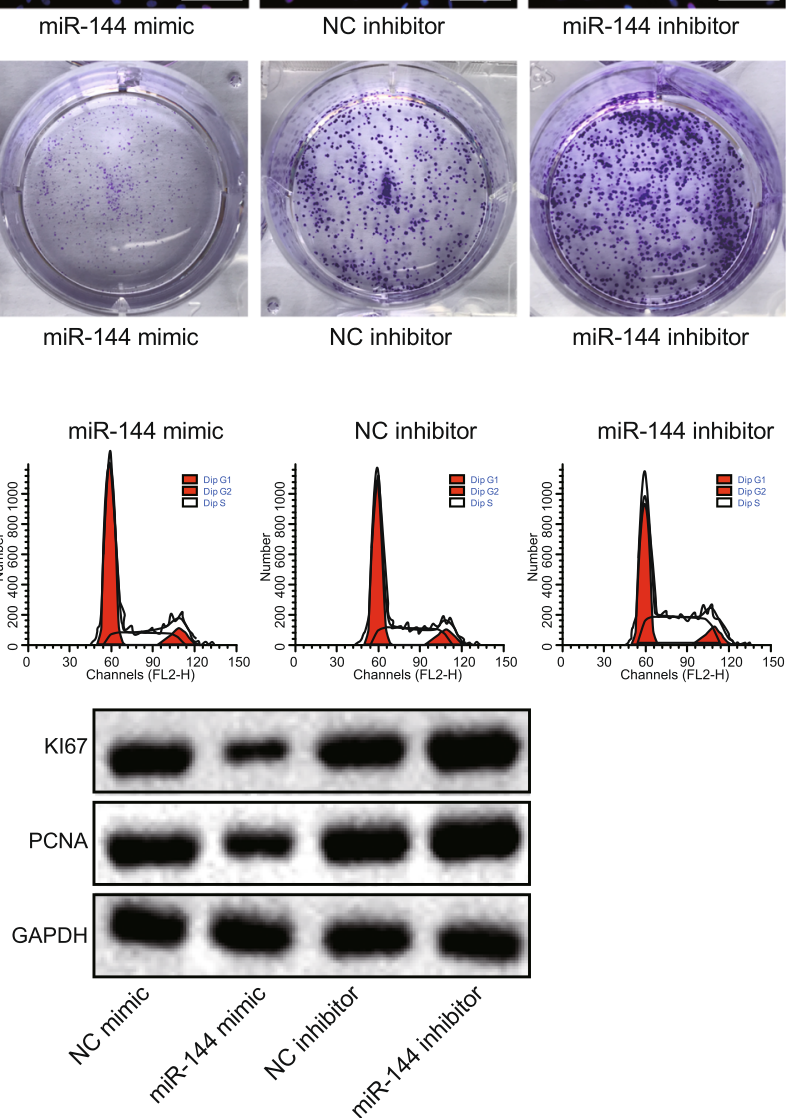
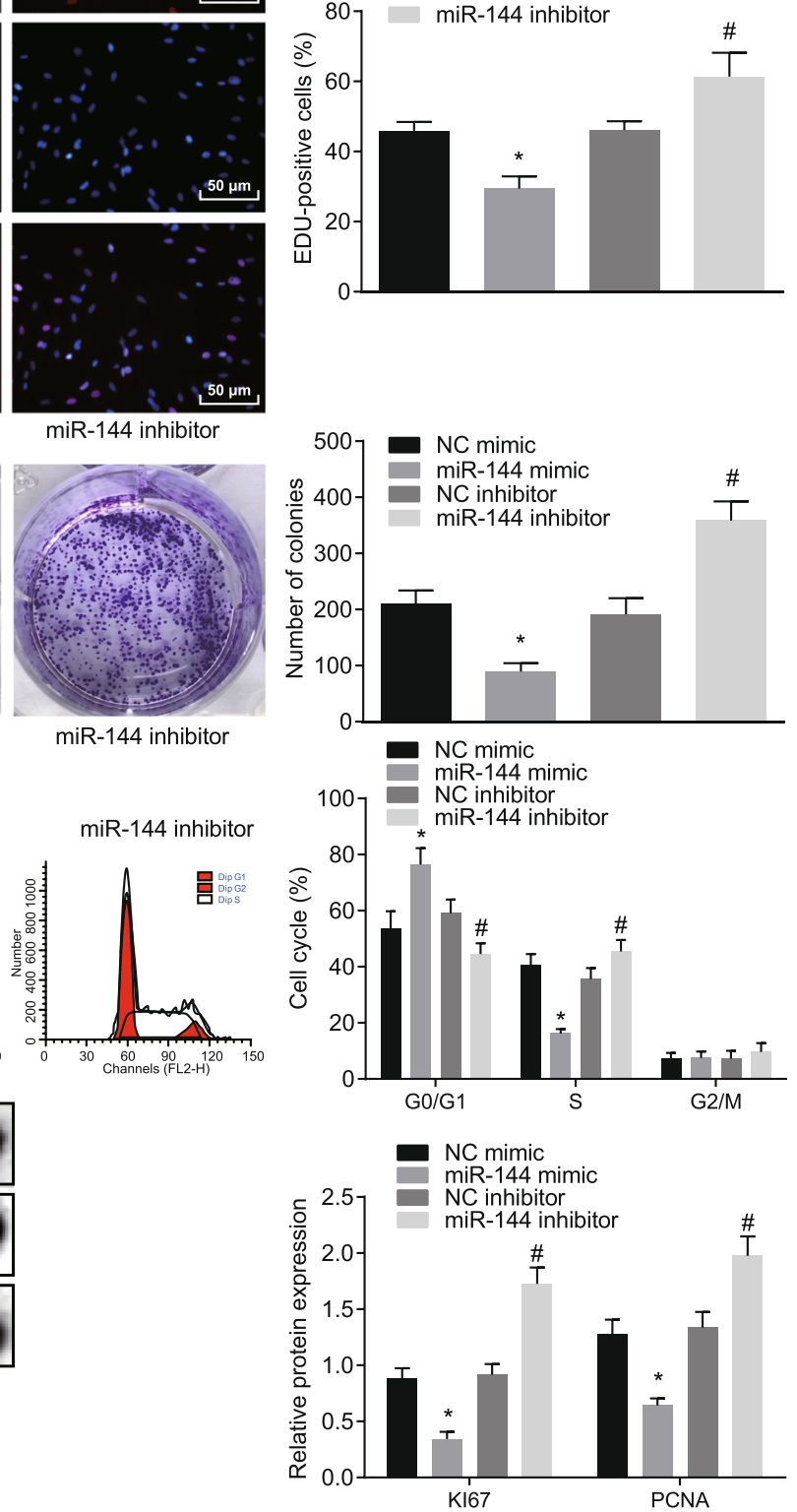

Fig. 4 Overexpression of miR-144 attenuates NSCLC cell proliferation and colony formation and reduces the number of S phase-arrested cells. a Cell proliferation detected by EdU assay $(\times 200)$. b Number of colony formation assessed by colony formation assay. c Cell cycle progression measured by flow cytometry. $\mathbf{d}$ The expression of proliferation-related factors determined by western blot analysis. ${ }^{*} p<0.05$ vs. A549 cells transfected with NC mimic. " $p<0.05$ vs. A549 cells transfected with NC inhibitor. The above measurement data were expressed as mean \pm standard deviation. Comparisons between two groups were analyzed by unpaired $t$ test. The experiment was repeated in triplicate

144 expression was appreciably elevated in response to miR-144 mimic treatment (versus NC mimic treatment) and decreased in response to miR-144 inhibitor treatment (versus NC inhibitor treatment) $(p<0.05)$. Hence, BMMSC-derived exosomes were isolated successfully.
BMMSC-derived exosomes restrained cell proliferation and colony formation in NSCLC cells through delivery of miR-144

BMMSC-derived exosomes labeled with Dil were cocultured with A549 cells. The exosome uptake of 


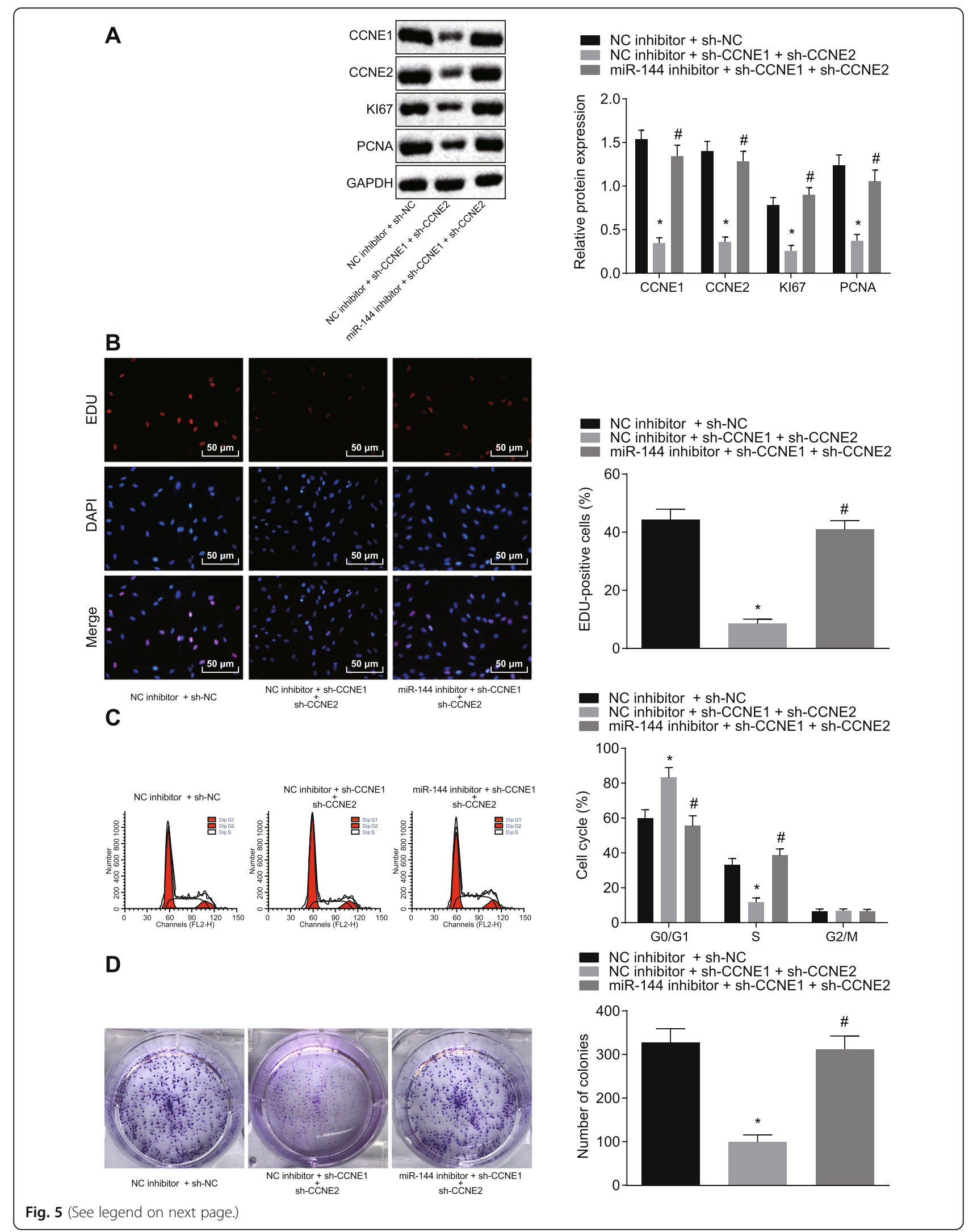


(See figure on previous page.)

Fig. 5 miR-144 impedes NSCLC cell proliferation and colony formation and reduces the number of S phase-arrested cells by downregulating CCNE1 and CCNE2. a The expression of proliferation-related factors detected by western blot analysis. $\mathbf{b}$ Cell proliferation assessed by EdU assay ( $\times 200$ ). c Cell cycle progression identified by flow cytometry. $\mathbf{d}$ Number of colony formation assessed by colony formation assay. ${ }^{*} p<0.05$ vs. A549 cells co-transfected with NC inhibitor + sh-NC. ${ }^{*} p<0.05$ vs. A549 cells co-transfected with NC inhibitor + sh-CCNE1 + sh-CCNE2 group. The above measurement data were expressed as mean \pm standard deviation. Data among multiple groups were analyzed by one-way ANOVA,

followed by a Tukey post hoc test. The experiment was repeated in triplicate

A549 cells under fluorescence microscopy suggested that after $48 \mathrm{~h}$ of co-culture, the exosome uptake of A549 cells was increased (Fig. 7a). RT-qPCR revealed that, compared with co-culture of Exo-NC mimic, miR-144 was highly expressed and CCNE1 and CCNE2 were poorly expressed in A549 cells after coculture with Exo-miR-144 mimic $(p<0.05)$ (Fig. 7b). In addition, in contrast to Exo-NC mimic results, cell proliferation and colony formation rate of A549 cells were decreased in response to Exo-miR-144 $(p<0.05)$ (Fig. 7c, d). Compared with Exo-NC mimic, the number of G0/G1 phase-arrested cells was increased, S phase-arrested cells were decreased, and expression of CCNE1, CCNE2, KI67, and PCNA was lower in A549 cells co-cultured with Exo-miR-144 (Fig. 7e, f).

\section{BMMSC-derived exosomes inhibited the growth of xenografted tumors in nude mice through delivery of miR-144 to NSCLC cells}

To prove further the inhibitory effect of BMMSCderived exosomes on tumor formation of A549 cells in vivo, A549 cells were injected subcutaneously into nude mice to establish the xenograft tumor model, while exosomes were injected through a tail vein. Then, the formation of xenografted tumor was observed and recorded. Compared with Exo-NCagomir treatment, the tumor volume and weight of xenograft tumors were reduced after injection of ExomiR-144 agomir $(p<0.05)$ (Fig. $8 \mathrm{a}-\mathrm{c})$. IHC revealed that positive rates of CCNE1, CCNE2, and KI67 were reduced in the xenografted tumor tissues upon the injection of Exo-miR-144 agomir compared with the injection of Exo-NC-agomir $(p<0.05)$ (Fig. 8d). Next, western blot analysis showed that expression of KI67 and PCNA in tumor tissues was downregulated in response to injection with Exo-miR-144 agomir, compared to findings with Eco-NC agomir $(p<0.05)$ (Fig. 8e).

\section{Discussion}

NSCLC has various pathological features [15]. NSCLC has a special high tumor mutation burden, which is defined as the number of non-synonymous coding mutations per megabase, especially in smokers [16]. MSC-derived exosomes have been shown to play essential roles in tumorigenesis, angiogenesis, and metastasis and can also present with a tumorsuppressing effect [17]. In the current study, we investigated the effect on NSCLC development of miR-144 shuttled from the BMMSC exosomes, finding that delivery of miR-144 via BMMSC-derived exosomes could downregulate CCNE1 and CCNE2 expression, thus attenuating NSCLC cell proliferation, colony formation, and cell cycle progression.

Our initial findings showed that CCNE1 and CCNE2 were highly expressed while miR-144 was poorly expressed in NSCLC tissues and cell lines. CCNE1 was reported to be repeatedly amplified and/ or upregulated in high-grade serous ovarian cancer [18]. The previous literature has confirmed that upregulation of CCNE1 occurs in human hepatocellular carcinomas [19]. High expression of CCNE2 in NSCLC tissues was also demonstrated in a previous report [6]. In addition, decreased miR-144 expression has been found in colorectal cancer tissues [20] and likewise in osteosarcoma cell lines and tissues [21]. In agreement with our findings, low expression miR-144 was revealed in lung adenocarcinoma tissues [22]. Moreover, CCNE1 and CCNE2 were targets of miR144 , which could be inversely regulated by miR-144. Similarly, CCNE1 and CCNE2 have been previously identified as direct targets of miR-144-5p; bladder cancer patients with high CCNE1 or CCNE2 expression have lower overall survival rate than patients with low expression [23], which suggested that downregulation of CCNE1 and CCNE2 inhibited bladder cancer cell proliferation. Consistent with our present results, downregulation of CCNE1 and CCNE2 could inhibit NSCLC cell proliferation, colony formation, and the number of $\mathrm{S}$ phase-arrested cells. Also, we find that miR-144 prevented cell proliferation and colony formation as well as S phase-arrest of NSCLC cells by downregulating CCNE1 and CCNE2. A previous literature proved that miR-144 delivery restrained growth and induced apoptosis of NSCLC cells [24]. Ki67 is a proliferation marker [25] and PCNA is protein needed for cell cycle progression from G1 to $S$ phase [26]. Our study also revealed that overexpression of miR-144 could decrease the expression of the markers Ki67 and PCNA. Taken together, the 


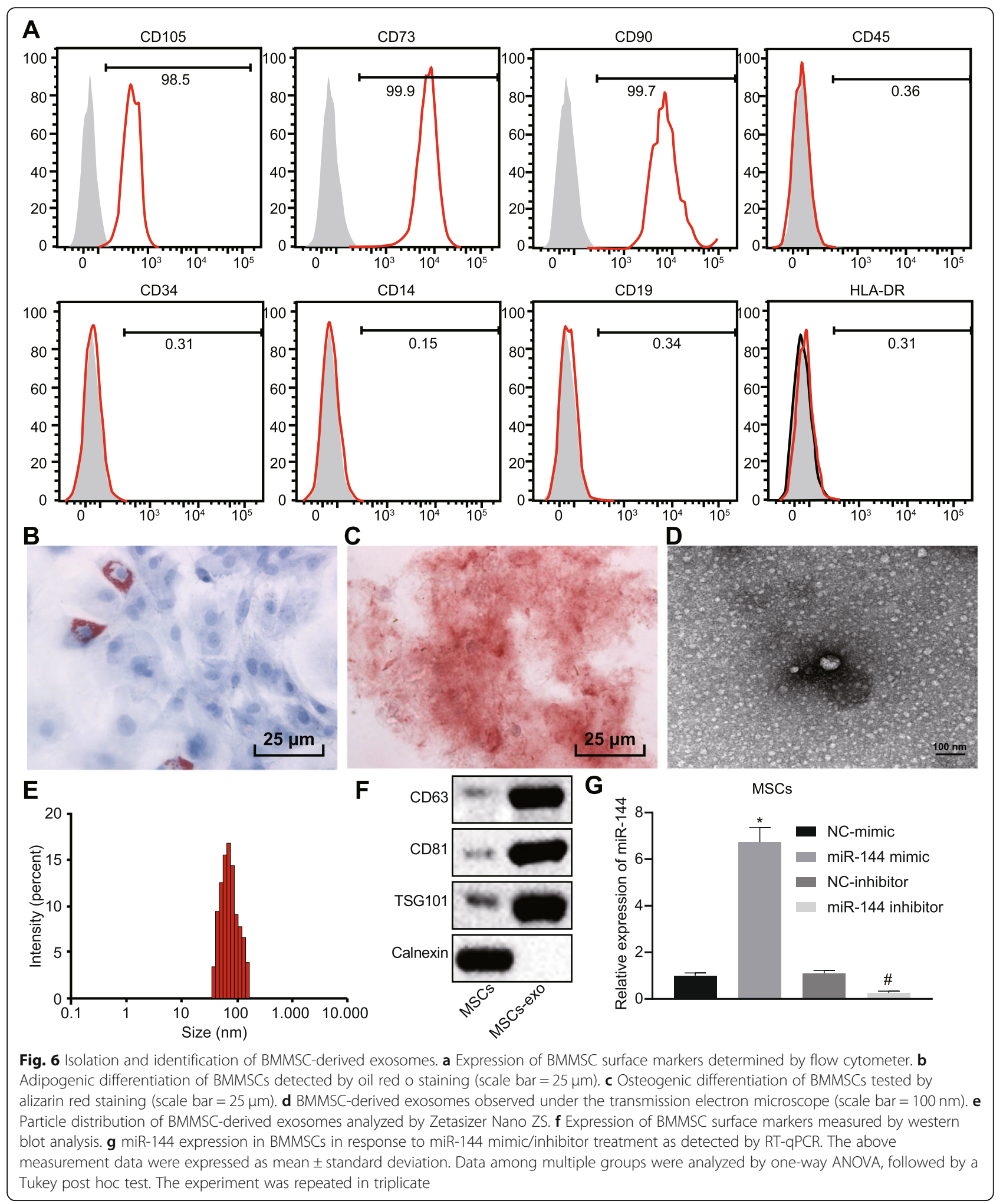

aforementioned findings suggest an inhibitory role of miR-144 in NSCLC cell biological functions, which is mediated via suppression of CCNE1 and CCNE2.
MSCs are a population of adult stem cells with autophagic and multilineage differentiation capabilities, including to chondrocytes, osteocytes, and 
A

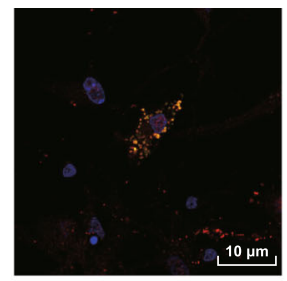

C
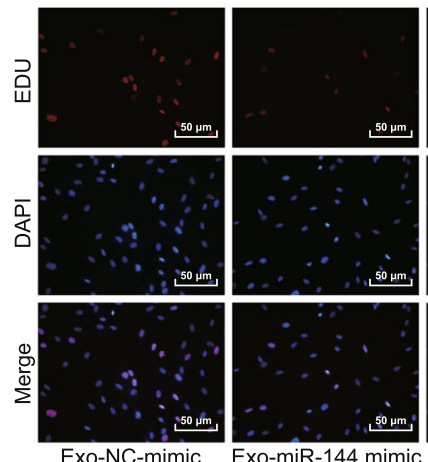

Exo-NC-mimic

D

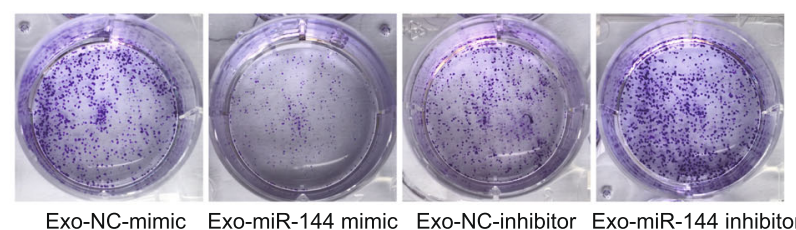

E

Exo-NC-mimic Exo-miR-144 mimic Exo-NC-inhibitor Exo-miR-144 inhibitor

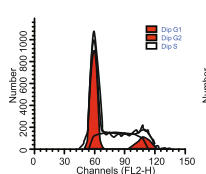

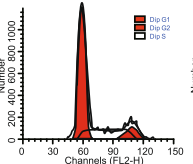
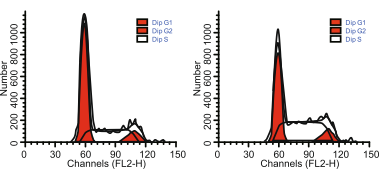

F

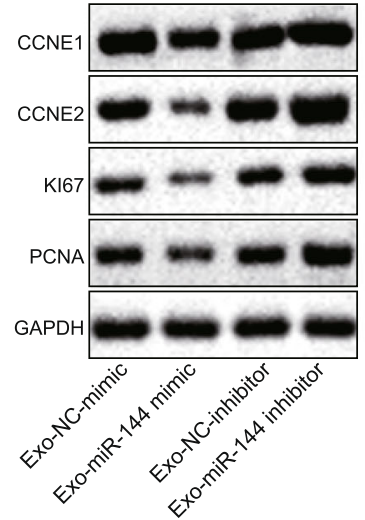

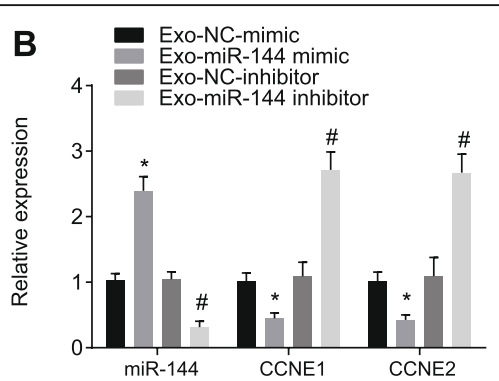
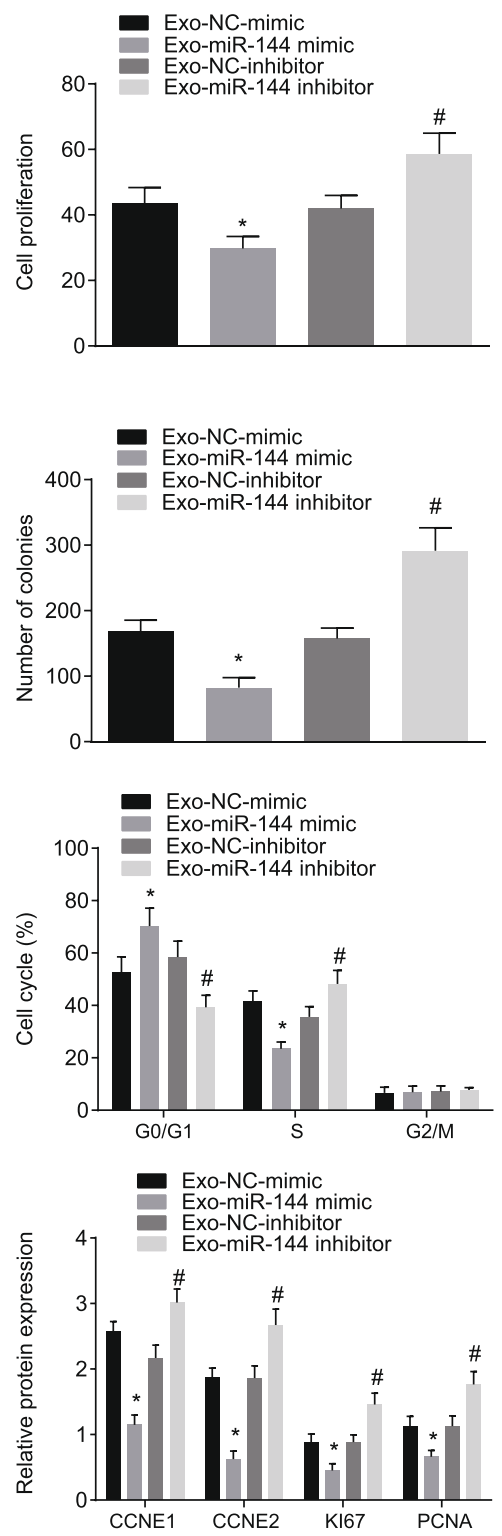

Fig. 7 (See legend on next page.) 
(See figure on previous page.)

Fig. 7 BMMSC-derived exosomes suppress cell proliferation and colony formation through delivering miR-144 to A549 cells. a Uptake of exosomes by A549 cells observed under a fluorescence microscope ( $\times 1000)$. b Relative expression of miR-144, CCNE1, and CCNE2 detected by RT-qPCR. c Cell proliferation assessed by EdU assay (× 200). $\mathbf{d}$ Number of colony formation identified by colony formation assay. e Cell cycle progression measured by flow cytometry. $\mathbf{f}$ Relative expression of proliferation related factors tested by western blot analysis. ${ }^{*} p<0.05$ vs. A549 cells transfected with Exo-NC mimic. ${ }^{p} p<0.05$ vs. A549 cells transfected with Exo-NC inhibitor. The above measurement data were expressed as mean \pm standard deviation. Comparisons between two groups were analyzed by unpaired $t$ test. The experiment was repeated in triplicate

adipocytes. The BMMSC secretome can inhibit the growth and promote apoptosis of NSCLC cells [27], and BMMSCs can be identified by their expression of certain surface markers. High expression of CD90 and CD105 and absent expression of CD34 were reported in MSCs in a previous study [28]. Consistently, we have identified that CD105 (76.99 $\pm 31.05 \%), C D 73$ $(96.43 \pm 3.88 \%)$, and $\mathrm{CD} 90(89.87 \pm 8.80 \%)$ were positively expressed, while CD45, CD34, and HLA-DR were negatively expressed in BMMSCs [29]. BMMSCs migrate to lung tumors, differentiate into lymphatic endothelial cells, and then participate in lymphangiogenesis [30]. In addition, our earlier work found that
BMMSCs can transfer miR-144 to NSCLC cells through transport in exosomes. Indeed, exosomes are not only important in intercellular communication, but can be also used as carriers of therapeutic genes and drugs [31]. miRNAs loaded into exosomes can be delivered to the recipient's niche cells, where they have a great impact on gene expression regulation [32]. A highly diverse population of miRNAs is observed in exosomes from human adipose mesenchymal stem cells, whose derived exosomal miRNAs are reportedly a critical factor for triggering antiproliferation signaling to ovarian cancer cells [33]. Moreover, exosomes containing certain miRNAs can

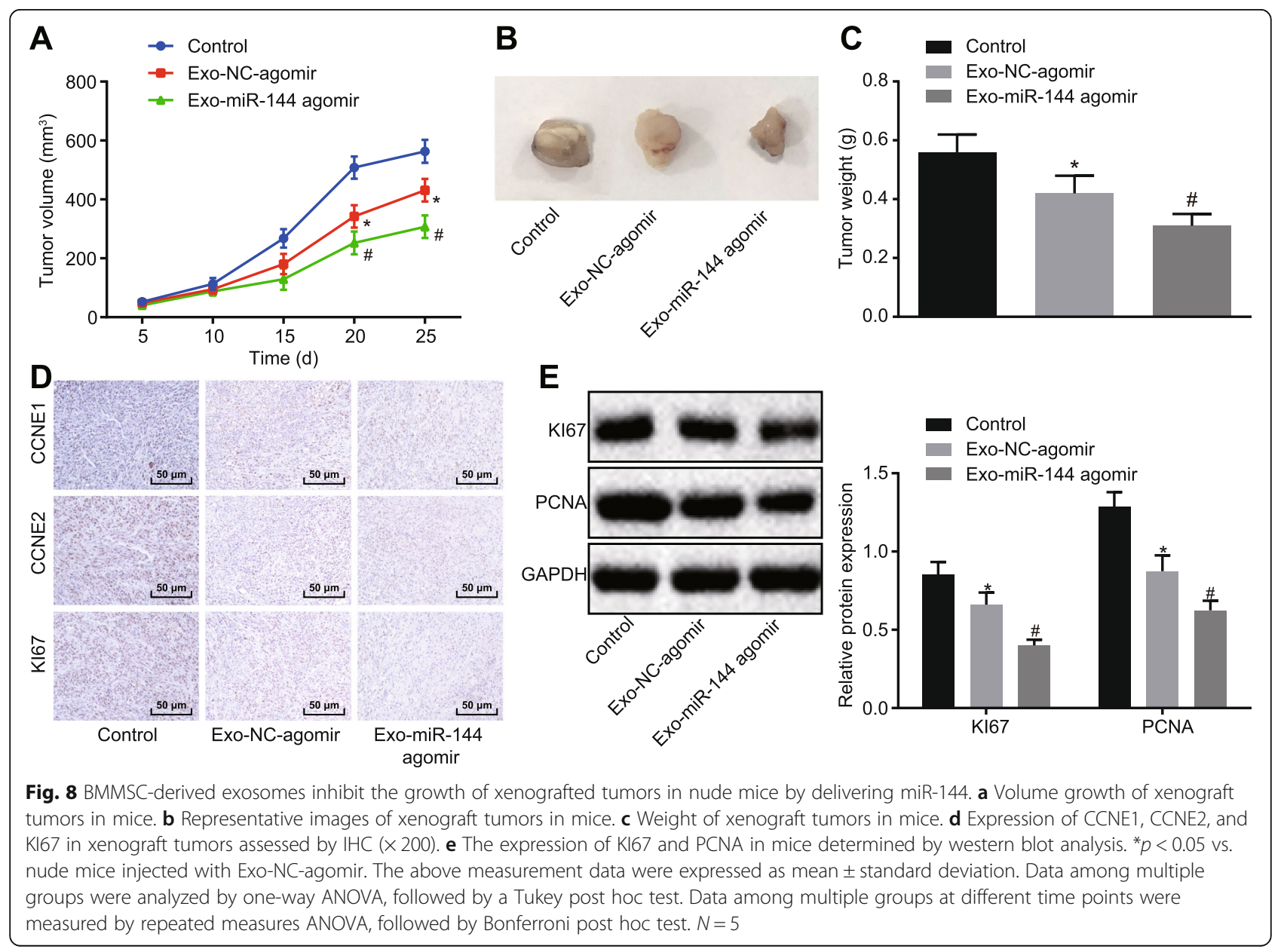




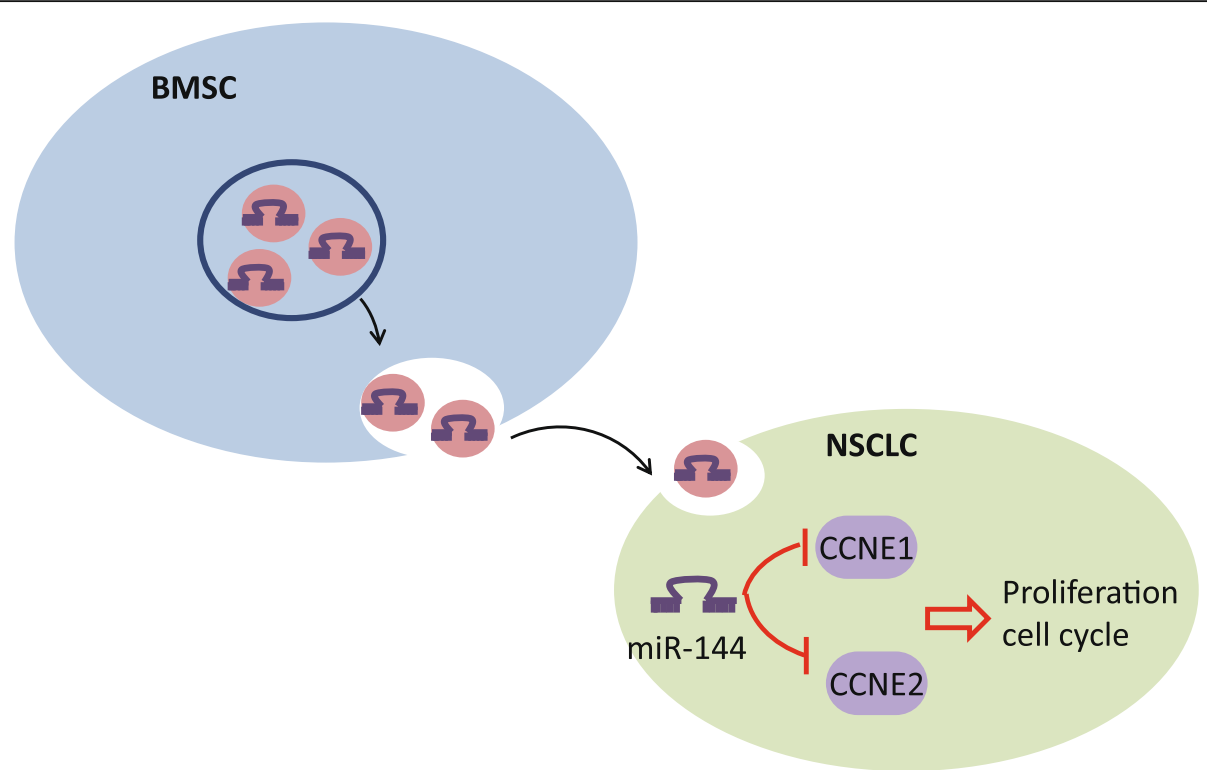

Fig. 9 Regulatory mechanism of BMMSC-derived exosomal miR-144 involved in NSCLC cell proliferation and cell cycle progression. BMMSCderived exosomal miR-144 impeded the proliferation and cell cycle of NSCLC cells by targeting CCNE1 and CCNE2

contribute to the delayed initiation and progression of chronic obstructive pulmonary disease (COPD) and are consequently widely used as diagnostic and prognostic biomarkers in COPD patients [34]. Thus, in keeping with a broad literature, we now report that BMMSC-derived exosomal miR-144 can impede NSCLC cell proliferation, colony formation, and ectopic tumor growth in nude mice.

\section{Conclusions}

BMMSC-derived exosomes carrying miR-144 can lower the expression of CCNE1 and CCNE2, thereby inhibiting progression of NSCLC (Fig. 9). The current study sheds new light on the potential of exosomes carrying miR-144 in impacting the development of NSCLC and on the use of miR-144 as new diagnostic and therapeutic biomarkers in the treatment of NSCLC.

\footnotetext{
Abbreviations

ANOVA: Analysis of variance; BMMSCs: Bone marrow-derived MSCs; CCNE1: Cyclin E1; DEGs: Differentially expressed genes; EdU: 5-ethynyl-2'deoxyuridine; FBS: Fetal bovine serum; FITC: Fluorescein isothiocyanate; GAPDH: Glyceraldehyde-3-phosphate dehydrogenase; HBE: Human bronchial epithelial; IgG: Immunoglobulin G; IHC: Immunohistochemistry; MEM: Minimum essential medium; miRs or miRNAs: microRNAs; MSCs: Mesenchymal stem cells; MUT: Mutant; NC: Negative control; NSCLC: Non-small cell lung cancer; PBS: Phosphate-buffered saline; PCNA: Proliferating cell nuclear antigen; PI: Propidium iodide; RTqPCR: Reverse transcription quantitative polymerase chain reaction; sh: Short hairpin; UTR: Untranslated region; WT: Wild type; ZYG11A: ZYG-11 family member A
}

\section{Acknowledgements}

We would like to give our sincere appreciation to the colleagues for their helpful comments and technical assistance on this article.

\section{Authors' contributions}

$Y L, D Z, L L, T X, Y Z, R M$, and JD designed the study. YL, DZ, and LL collated the data, carried out the data analyses, and produced the initial draft of the manuscript. TX, YZ, RM, and JD contributed to the drafting of the manuscript. All authors have read and approved the final submitted manuscript.

\section{Funding}

Not applicable.

\section{Availability of data and materials}

The datasets generated/analyzed during the current study are available.

\section{Ethics approval and consent to participate}

This experiment was approved by the Ethics Committee of Cancer Hospital of China Medical University. All participants enrolled in the study signed informed written consent documents, and the experiment procedures were in line with the Declaration of Helsinki. All animal experiments were in strict accordance with the recommendations in the Guide for the Care and Use of Laboratory Animals of the National Institutes of Health.

\section{Consent for publication}

Not applicable.

\section{Competing interests}

The authors declare that they have no competing interests.

\section{Author details}

${ }^{1}$ Medical Oncology Department of Thoracic Cancer (2), Cancer Hospital of China Medical University, Liaoning Cancer Hospital \& Institute, No. 44, Xiaoheyan Road, Dadong District, Shenyang 110042, Liaoning Province, People's Republic of China. ${ }^{2}$ Department of Thyroid Surgery, The First Affiliated Hospital of China Medical University, Shenyang 110001, People's Republic of China. ${ }^{3}$ Department of Pathology, The First Affiliated Hospital and College of Basic Medical Science, China Medical University, No. 155, 
Nanjing North Street, Heping District, Shenyang 110001, Liaoning Province, People's Republic of China.

Received: 5 August 2019 Revised: 6 December 2019 Accepted: 4 February 2020 Published online: 26 February 2020

\section{References}

1. Coudray N, et al. Classification and mutation prediction from non-small cell lung cancer histopathology images using deep learning. Nat Med. 2018; 24(10):1559-67.

2. Davidson MR, Gazdar AF, Clarke BE. The pivotal role of pathology in the management of lung cancer. J Thorac Dis. 2013;5(Suppl 5):S463-78.

3. Bao Q, et al. Mesenchymal stem cell-based tumor-targeted gene therapy in gastrointestinal cancer. Stem Cells Dev. 2012;21(13):2355-63.

4. Han Z, et al. miR-497 and miR-34a retard lung cancer growth by coinhibiting cyclin E1 (CCNE1). Oncotarget. 2015;6(15):13149-63.

5. Wang $X$, et al. ZYG11A serves as an oncogene in non-small cell lung cancer and influences CCNE1 expression. Oncotarget. 2016;7(7):8029-42.

6. Chen $D$, et al. MicroRNA-30d-5p inhibits tumour cell proliferation and motility by directly targeting CCNE2 in non-small cell lung cancer. Cancer Lett. 2015;362(2):208-17.

7. Chuanyin $\mathrm{L}$, et al. The association between polymorphisms in microRNA genes and cervical cancer in a Chinese Han population. Oncotarget. 2017; 8(50):87914-27.

8. Mairinger FD, et al. Different micro-RNA expression profiles distinguish subtypes of neuroendocrine tumors of the lung: results of a profiling study. Mod Pathol. 2014;27(12):1632-40.

9. Gao F, et al. Regulation of activating protein-4-associated metastases of non-small cell lung cancer cells by miR-144. Tumour Biol. 2015. https://doi. org/10.1007/s13277-015-3866-4.

10. Li X, et al. Challenges and opportunities in exosome research-perspectives from biology, engineering, and cancer therapy. APL Bioeng. 2019;3(1): 011503

11. Shi B, et al. Bone marrow mesenchymal stem cell-derived exosomal miR-21 protects C-kit+ cardiac stem cells from oxidative injury through the PTEN/ PI3K/Akt axis. PLoS One. 2018;13(2):e0191616.

12. Bhat A, Sharma A, Bharti AC. Upstream Hedgehog signaling components are exported in exosomes of cervical cancer cell lines. Nanomedicine (Lond). 2018;13(17):2127-38.

13. Bing Z, et al. Effect of mechanical stretch on the expressions of elastin, LOX and Fibulin-5 in rat BMSCs with ligament fibroblasts co-culture. Mol Bio Rep. 2012;39(5):6077-85.

14. Caldon CE, Musgrove EA, et al. Cell Div. 2010;5:2.

15. Chen Z, et al. Non-small-cell lung cancers: a heterogeneous set of diseases. Nat Rev Cancer. 2014;14(8):535-46.

16. Herbst RS, Morgensztern D, Boshoff C. The biology and management of non-small cell lung cancer. Nature. 2018;553(7689):446-54.

17. Vakhshiteh F, Atyabi F, Ostad SN. Mesenchymal stem cell exosomes: a twoedged sword in cancer therapy. Int J Nanomedicine. 2019;14:2847-59.

18. Karst AM, et al. Cyclin E1 deregulation occurs early in secretory cell transformation to promote formation of fallopian tube-derived high-grade serous ovarian cancers. Cancer Res. 2014;74(4):1141-52.

19. Nault JC, et al. Recurrent AAV2-related insertional mutagenesis in human hepatocellular carcinomas. Nat Genet. 2015;47(10):1187-93.

20. Iwaya T, et al. Downregulation of miR-144 is associated with colorecta cancer progression via activation of mTOR signaling pathway. Carcinogenesis. 2012;33(12):2391-7.

21. Wang W, Zhou X, Wei M. MicroRNA-144 suppresses osteosarcoma growth and metastasis by targeting ROCK1 and ROCK2. Oncotarget. 2015;6(12): 10297-308.

22. Ma T, et al. Tumor-promoting activity of long noncoding RNA LINC00466 in lung adenocarcinoma via miR-144-regulated HOXA10 axis. Am J Pathol. 2019;189(11):2154-70

23. Matsushita R, et al. Tumour-suppressive microRNA-144-5p directly targets CCNE1/2 as potential prognostic markers in bladder cancer. Br J Cancer. 2015:113(2):282-9.

24. Zha W, et al. Roles of Mir-144-ZFX pathway in growth regulation of nonsmall-cell lung cancer. PLoS One. 2013:8(9):e74175.

25. Galimberti F, et al. Targeting the cyclin E-Cdk-2 complex represses lung cancer growth by triggering anaphase catastrophe. Clin Cancer Res. 2010; 16(1):109-20.
26. Pal HC, et al. Delphinidin reduces cell proliferation and induces apoptosis of non-small-cell lung cancer cells by targeting EGFR/NEGFR2 signaling pathways. PLoS One. 2013;8(10):e77270.

27. Attar-Schneider $\mathrm{O}$, et al. Secretome of human bone marrow mesenchymal stem cells: an emerging player in lung cancer progression and mechanisms of translation initiation. Tumour Biol. 2016;37(4):4755-65.

28. Barberini DJ, et al. Equine mesenchymal stem cells from bone marrow, adipose tissue and umbilical cord: immunophenotypic characterization and differentiation potential. Stem Cell Res Ther. 2014;5(1):25.

29. Vereb Z, et al. Role of human corneal stroma-derived mesenchymal-like stem cells in corneal immunity and wound healing. Sci Rep. 2016;6:26227.

30. Zhou XM, et al. Bone marrow derived mesenchymal stem cells involve in the lymphangiogenesis of lung cancer and Jinfukang inhibits the involvement in vivo. J Cancer. 2017;8(10):1786-94.

31. Zhang D, et al. Enrichment of selective miRNAs in exosomes and delivery of exosomal miRNAs in vitro and in vivo. Am J Physiol Lung Cell Mol Physiol. 2017;312(1):L110-L21.

32. Yan W, et al. Cancer-cell-secreted exosomal miR-105 promotes tumour growth through the MYC-dependent metabolic reprogramming of stromal cells. Nat Cell Biol. 2018;20(5):597-609.

33. Reza A, et al. Human adipose mesenchymal stem cell-derived exosomalmiRNAs are critical factors for inducing anti-proliferation signalling to A2780 and SKOV-3 ovarian cancer cells. Sci Rep. 2016;6:38498

34. Salimian J, et al. Chronic obstructive pulmonary disease: microRNAs and exosomes as new diagnostic and therapeutic biomarkers. J Res Med Sci. 2018:23:27.

\section{Publisher's Note}

Springer Nature remains neutral with regard to jurisdictional claims in published maps and institutional affiliations.

Ready to submit your research? Choose BMC and benefit from:

- fast, convenient online submission

- thorough peer review by experienced researchers in your field

- rapid publication on acceptance

- support for research data, including large and complex data types

- gold Open Access which fosters wider collaboration and increased citations

- maximum visibility for your research: over $100 \mathrm{M}$ website views per year

At BMC, research is always in progress.

Learn more biomedcentral.com/submissions 